\title{
Performance Improvement of a Micro-stimulus Electrode for Retinal Prosthesis by Introducing a High-Performance Material and a Three-Dimensional Structure
}

\author{
Takumi Fujisawa, Toshihiko Noda, ${ }^{*}$ Megumi Hayashi, Ryo Kobe, Hiroyuki Tashiro, ${ }^{1}$ \\ Hiroaki Takehara, Kiyotaka Sasagawa, Takashi Tokuda, Chung-Yu Wu, ${ }^{2}$ and Jun Ohta \\ Nara Institute of Science and Technology, 8916-5 Takayama, Ikoma, Nara 630-0192, Japan \\ ${ }^{1}$ Kyushu University, 3-1-1 Maidashi, Higashi-ku, Fukuoka, Fukuoka 812-8582, Japan \\ ${ }^{2}$ National Chiao Tung University, 1001 University Road, Hsinchu 30010, Taiwan
}

(Received May 2, 2016; accepted August 9, 2016)

Keywords: retinal prosthesis, stimulus electrode, iridium oxide, three-dimensional electrode, charge injection capacity

High-performance electrodes intended for retinal prosthesis were fabricated and evaluated. Iridium oxide $\left(\mathrm{IrO}_{x}\right)$ was introduced as a high-performance material. A three-dimensional (3D) structure was also introduced to enlarge the electrode's surface area. We tried to improve the electrode performance by combining these approaches, even if the electrode was miniaturized. The effectiveness of $\mathrm{IrO}_{x}$ was demonstrated through electrochemical evaluation by comparing it with Pt. $\mathrm{IrO}_{x}$ showed 1.6-6 times higher performance for the injection of stimulus pulse current than Pt. The performance of the 3D electrode compared with a planar electrode was also evaluated. Accordingly, the 3D electrode showed 2-4 times higher performance than the planar electrode by surface area enlargement. An ex vivo validation of the stimulus performance was conducted to demonstrate its practical use. A fabricated electrode was implanted in an extracted pig eyeball and the electrochemical performance was evaluated. The fabricated electrode showed sufficient performance of the retinal stimulation, with a high margin of safety. The proposed approach was successfully demonstrated as a stimulus electrode candidate for use in next-generation retinal prosthesis.

\section{Introduction}

Retinal prosthesis systems, which partially restore the vision of blind patients, have recently been developed. Some of these systems have obtained medical approval in Europe and/or the United States. ${ }^{(1)}$ A retinal prosthesis for practical use is currently in its startup phase. However retinal prosthesis systems need to be improved. One such need is improvement of the restored sight resolution. Increasing the number of stimulus electrodes for high density stimulation of the retina is required to achieve high-resolution restoration of sight.

In a retinal prosthesis, phosphene is evoked by electrical stimulation of the retina. Three main methods are used for retinal stimulation, namely, epi-retinal stimulation, sub-retinal stimulation, and suprachoroidal-transretinal stimulation (STS). The difference among these methods is the

*Corresponding author: e-mail: t-noda@ms.naist.jp 
implantation positions of the stimulus electrodes. The material and shape of the stimulus electrodes are frequently evaluated for safely, effectively, and densely stimulating retinal tissue. The number of stimulus electrodes must be increased to realize high-density stimulation. However, wiring a huge number of electrodes and miniaturizing them is difficult. We propose a smart electrode for the STS as one solution to such wiring difficulties. This solution achieves control of multiple electrodes using little wiring by introducing complementary metal-oxide semiconductor (CMOS) microchips. ${ }^{(2-5)}$ A number of trials, including combination with a CMOS microchip ${ }^{(6)}$ or electrode fabrication on a CMOS microchip directory, ${ }^{(7)}$ have been performed for epi-retinal and sub-retinal stimulations. These trials aim to increase the number of stimulus electrodes. Introducing CMOS microchips is an important strategy for increasing the number of electrodes.

Meanwhile, developing the small high-performance electrodes required to increase the number of stimulus electrodes is an issue that needs to be resolved. The requirements for the stimulus electrodes are as follows:

- capability to evoke the phosphene;

- high charge injection capacity (CIC), which is the capability of electrical stimulation without tissue damage;

—non-toxicity and biocompatibility; and

- high durability under chronic implantation.

In the case of electrode miniaturization, the total capacity of the charge injection decreases proportionally to the decrease of the electrode surface area, because the CIC per unit surface area is not affected without material change. Meanwhile, the threshold only slightly decreases, even if the stimulus electrode is miniaturized, because of the non-linear relationship between the electrode size and the threshold current of stimulation. Therefore, a reduction of CIC is caused by the electrode miniaturization. Consequently, safe stimulation of the retina becomes difficult.

Enlarging the geometric and/or effective surface area of the electrode is one approach to maintaining the CIC, even in a miniaturized electrode. Several methods of enlarging the effective surface area have been reported. These include surface coating by Pt-black ${ }^{(8)}$ and porous surface fabrication by laser processing. ${ }^{(9,10)}$ Another approach is the utilization of a high-performance material that shows a high CIC. High-performance metallic materials, such as iridium oxide $\left(\operatorname{IrO}_{x}\right)^{(11,12)}$ and titanium nitride ${ }^{(13)}$ have been reported as electrode material candidates. Some organic materials, such as poly $\left(3,4\right.$-ethylenedioxythiophene) (PEDOT) ${ }^{(14)}$ have also been included. $\mathrm{IrO}_{x}$ is a nontoxic material expected to show high CIC, and which can be fabricated using various methods. The electrode properties of $\mathrm{IrO}_{x}$ show a wide variety according to fabrication methods and process parameters. ${ }^{(15-19)}$ We also discuss the use of $\mathrm{IrO}_{x}$ as a stimulus electrode for a retinal prosthesis. We fabricate an $\mathrm{IrO}_{x}$ electrode using the sputtering method because of its compatibility with the CMOS process. $^{(20,21)}$

This study proposes a combination of the two above-mentioned approaches to fabricate highperformance and miniaturized electrodes for retinal stimulation by enlarging the surface area using a three-dimensional (3D) structure, and surface coating with $\mathrm{IrO}_{x}$. A fabrication process is considered, and 3D $\mathrm{IrO}_{x}$ electrodes are fabricated. The performance of the fabricated electrodes is evaluated using electrochemical measurements. The effectiveness of introducing the $3 \mathrm{D}$ structure and the high-performance material is quantitatively evaluated by comparing the performance with planar and Pt electrodes. An ex vivo experiment is also performed using an extracted pig eyeball to compare the evaluation results with a phantom. 


\section{Electrode Fabrication}

Three types of electrodes are fabricated in this study. The first one is a square planar $\operatorname{IrO}_{x}$ electrode with $65 \mu \mathrm{m}$ sides (geometric surface area: $4.23 \times 10^{-5} \mathrm{~cm}^{2}$ ). The second one is a bumpshaped 3D $\mathrm{IrO}_{x}$ electrode, which has a height of $92 \mu \mathrm{m}$ and a diameter of $50 \mu \mathrm{m}$. The geometric surface area of the 3D electrode can be approximated as $1.55 \times 10^{-4} \mathrm{~cm}^{2}$. The third one, which is used as a reference, is a square planar Pt electrode with $65 \mu \mathrm{m}$ sides. All electrodes are fabricated on CMOS microchips with $65 \mu \mathrm{m}$ square aluminum electrodes. The Al electrodes are directly connected to bonding pads for external connection.

Figure 1 shows the fabrication process of the planar electrode. A photoresist (AZ 5214E, Merck Performance Materials) is first spin-coated on the microchip and patterned as the electrode area. Figure 1(b) shows that the lateral profile of the photoresist for the lift-off process is an inverted $\mathrm{V}$ shape. The $\mathrm{Al}$ electrode surface is then cleaned by Ar plasma in the reverse sputtering process. An $\mathrm{IrO}_{x}$ film $(1 \mu \mathrm{m})$ is subsequently deposited using the reactive sputtering process with $\mathrm{O}_{2}$ gas after Ti adhesive layer $(200 \mathrm{~nm})$ deposition. Table 1 shows the sputtering process parameters. Each parameter value is an optimized value, as reported in our previous work. ${ }^{(20,21)}$ The film-deposited microchip is dipped into the photoresist remover (AZ Remover 100, Merck performance Materials)

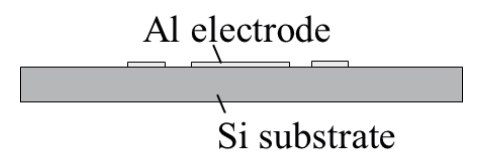

(a)

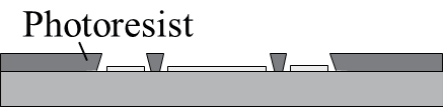

(b)

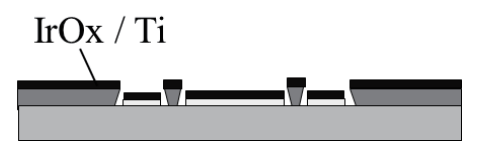

(c)

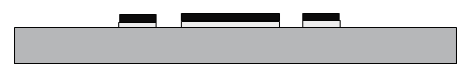

(d)

Fig. 1. Fabrication process of the planar electrode. (a) Initial state. (b) Photoresist patterning. (c) Ti and $\mathrm{IrO}_{x}$ sputtering. (d) Lift-off.

Table 1

Sputtering parameters.

\begin{tabular}{lcccc}
\hline & Reverse sputtering & $\mathrm{Ti}$ & $\mathrm{IrO}_{x}$ & $\mathrm{Pt}$ \\
\hline Gas & $\mathrm{Ar}$ & $\mathrm{Ar}$ & $\mathrm{O}_{2}$ & $\mathrm{Ar}$ \\
Gas flow rate & $10 \mathrm{sccm}$ & $20 \mathrm{sccm}$ & $10 \mathrm{sccm}$ & $20 \mathrm{sccm}$ \\
Pressure & $1.0 \mathrm{~Pa}$ & $0.6 \mathrm{~Pa}$ & $1.0 \mathrm{~Pa}$ & $0.6 \mathrm{~Pa}$ \\
Power & $\mathrm{RF} 50 \mathrm{~W}$ & $\mathrm{DC} 100 \mathrm{~W}$ & $\mathrm{RF} 200 \mathrm{~W}$ & $\mathrm{RF} 200 \mathrm{~W}$ \\
Time & $1 \mathrm{~min}$ & $5 \mathrm{~min}$ & $60 \mathrm{~min}$ & $10 \mathrm{~min}$ \\
\hline
\end{tabular}


at $80^{\circ} \mathrm{C}$. The film is then patterned by the lift-off process as shown in Fig. 1(d). Figure 2 shows the micrograph of the fabricated planar electrode. Instead of $\mathrm{IrO}_{x}, \mathrm{Pt}$ is deposited for Pt planar electrode fabrication. Table 1 also shows the sputtering parameters of $\mathrm{Pt}$.

Figure 3 shows the fabrication process of the 3D electrode. A Au stud bump is formed on the Al electrode after photoresist patterning with the $3 \mathrm{D}$ electrode. $\operatorname{IrO}_{x}$ is then deposited on the stud bump and patterned, using the same process flow as that in the planar electrode. Figure 4(a) shows the optical micrograph of the fabricated 3D electrode. Figure 4(b) also shows a SEM image of the $3 \mathrm{D}$ electrodes with a $65^{\circ}$ tilt angle.

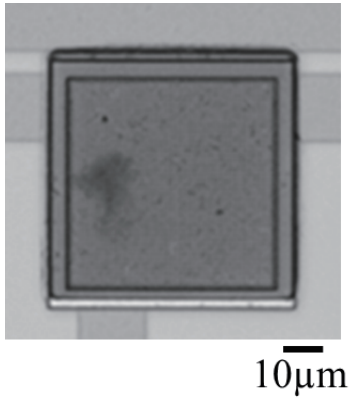

Fig. 2. Micrograph of the fabricated planar electrode.

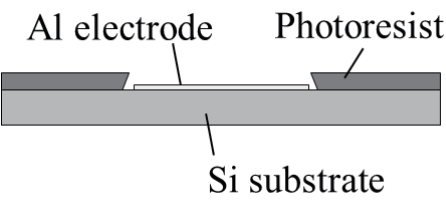

(a)

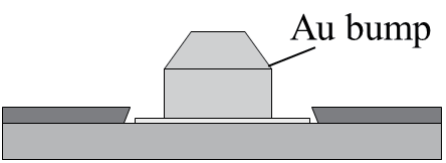

(b)

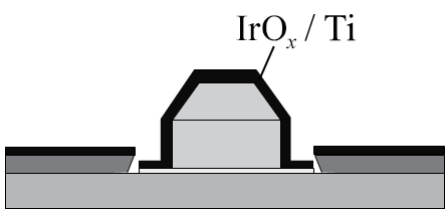

(c)

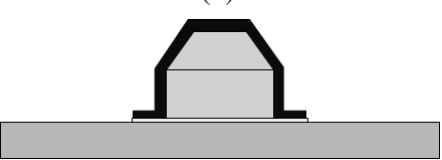

(d)

Fig. 3. Fabrication process of the 3D electrode. (a) Photoresist patterning. (b) Stud bump formation. (c) Ti and $\mathrm{IrO}_{x}$ coating. (d) Lift-off.

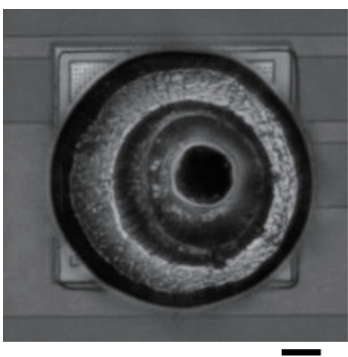

$1 \overline{0 \mu \mathrm{m}}$

(a)

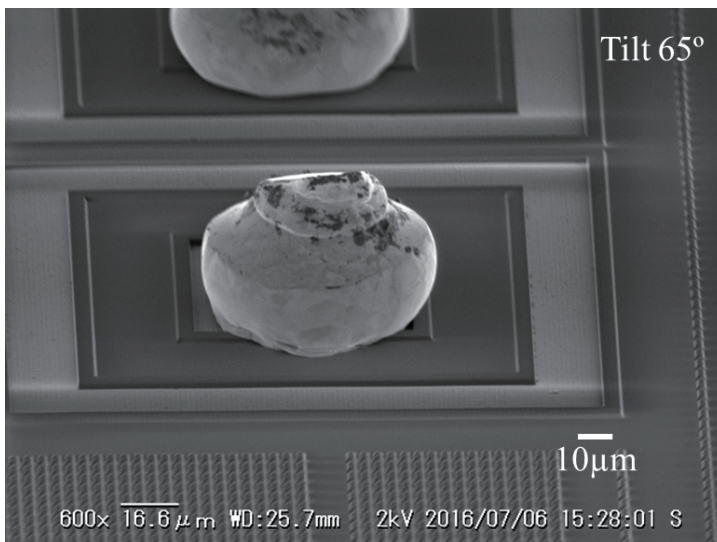

(b)

Fig. 4. Fabricated 3D electrode. (a) Optical micrograph. (b) SEM image with $65^{\circ}$ tilt angle. 


\section{Basic Characterization of the Electrodes}

Electrochemical evaluations of the fabricated electrodes were performed to measure the CIC properties under the both in vitro and ex vivo situations. In vitro evaluation is suitable for discussion of the fundamental electrochemical properties under ideal conditions. Accordingly, for the in vitro evaluation, $0.01 \mathrm{M}$ phosphate buffered saline (PBS) is used as a phantom to simulate the implantation environment of the electrode in biological tissue. On the other hand, ex vivo evaluation is also important to evaluate the electrode performance after implantation into biological tissue to determine its effectiveness in practical use.

In this study, two kinds of electrochemical evaluation were performed. Cyclic voltammetry (CV) measurement is performed to estimate the charge storage capacity (CSC), which is one of the indicators of electrode performance in the low-frequency domain.(22) The CIC, which is the maximum injectable charge without tissue damage, can be measured by measuring the transient electrode potential under stimulus current pulse application.

\subsection{Experimental setup of the in vitro electrochemical measurement}

\subsubsection{CV measurement}

Figure 5 shows the experimental setup of the $\mathrm{CV}$ measurement, where a conventional threeelectrode method is used. A Pt wire is utilized as a counter electrode, while an $\mathrm{Ag} / \mathrm{AgCl}$ electrode with saturated $\mathrm{KCl}$ is employed as a reference electrode. The fabricated electrode, which is a work electrode, is dipped into the PBS. The three electrodes are connected to a potentiostat (PGSTAT 204, Metrohm). The scanning ranges of the work electrode potential are $-0.65-0.8 \mathrm{~V}$ and $-0.6-$ $1.05 \mathrm{~V}$ for $\mathrm{IrO}_{x}$ and $\mathrm{Pt}$, respectively. The scanning rate of the potential is $50 \mathrm{mV} / \mathrm{s}$.

\subsubsection{Measurement of the transient electrode potential}

Figure 6 shows the experimental setup of the transient potential measurement. The threeelectrode method is used, similar to that in the CV measurement. The current pulses, which are generated by an electronic stimulator, are applied between the counter and work electrodes. The electrode potential versus the reference electrode is measured by an electrometer (PGSTAT204, Metrohm Autolab). The electronic stimulator generates biphasic pulses with the following pulse

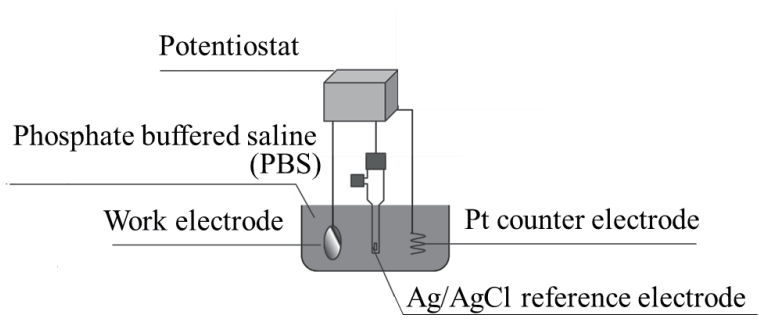

Fig. 5. Experimental setup of the CV measurement.

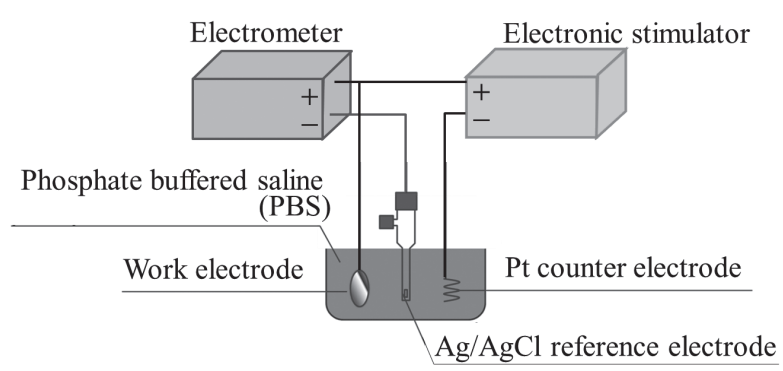

Fig. 6. Experimental setup of the transient potential measurement. 
parameters; pulse width: $0.5 \mathrm{~ms}$, inter-pulse duration: $0.2 \mathrm{~ms}$, and repetition frequency: $50 \mathrm{kHz}$. The pulse current is gradually increased from a lower value until the electrode potential reaches the limit of the potential window of water.

\subsection{Comparison of material difference properties}

Figure 7 shows the results of the $\mathrm{CV}$ measurement of the $\mathrm{IrO}_{x}$ and $\mathrm{Pt}$ planar electrodes. The CSC value can be calculated by integrating the closed area of a CV curve.(22) Table 2 shows the calculated CSC value. The CSC value of the $\mathrm{IrO}_{x}$ electrode is 12 times larger than that of the $\mathrm{Pt}$ electrode.

Figure 8 shows the results of the transient potential measurement of the $\mathrm{IrO}_{x}$ electrode. The electrode potential in the anodic-first (AF) biphasic pulse reaches a positive limit of the potential window of water (i.e., $0.8 \mathrm{~V}$ ) with a $60 \mu \mathrm{A}$ pulse. From the results, the maximum allowable current without electrolysis can be estimated as $60 \mu \mathrm{A}$. This finding indicates that the charge amount is 0.03 $\mu \mathrm{C}$ in a $0.5 \mathrm{~ms}$ pulse. The geometric surface area of the electrode is $4.23 \times 10^{-5} \mathrm{~cm}^{2}$. Therefore, the unit area CIC of the electrode is $0.71 \mathrm{mC} / \mathrm{cm}^{2}$. Similarly, the maximum allowable current in the cathodic-first (CF) pulse is $80 \mu \mathrm{A}$, and the unit area CIC is $0.95 \mathrm{mC} / \mathrm{cm}^{2}$. Table 3 shows the measured CICs of the Pt electrode. The CDCs of the $\mathrm{IrO}_{x}$ electrode are six times larger with the AF pulse and 1.6 times larger with the CF pulse than those of the Pt electrode.

The results of the CSC and CIC evaluations illustrate that $\mathrm{IrO}_{x}$ shows a higher performance than Pt. The CSC improvement by material change is larger than the CIC improvement. The $\mathrm{CIC}$ includes the electrode performance in the high-frequency domain because the pulse current used for the CIC measurement has higher frequency components. The difference of the electrode

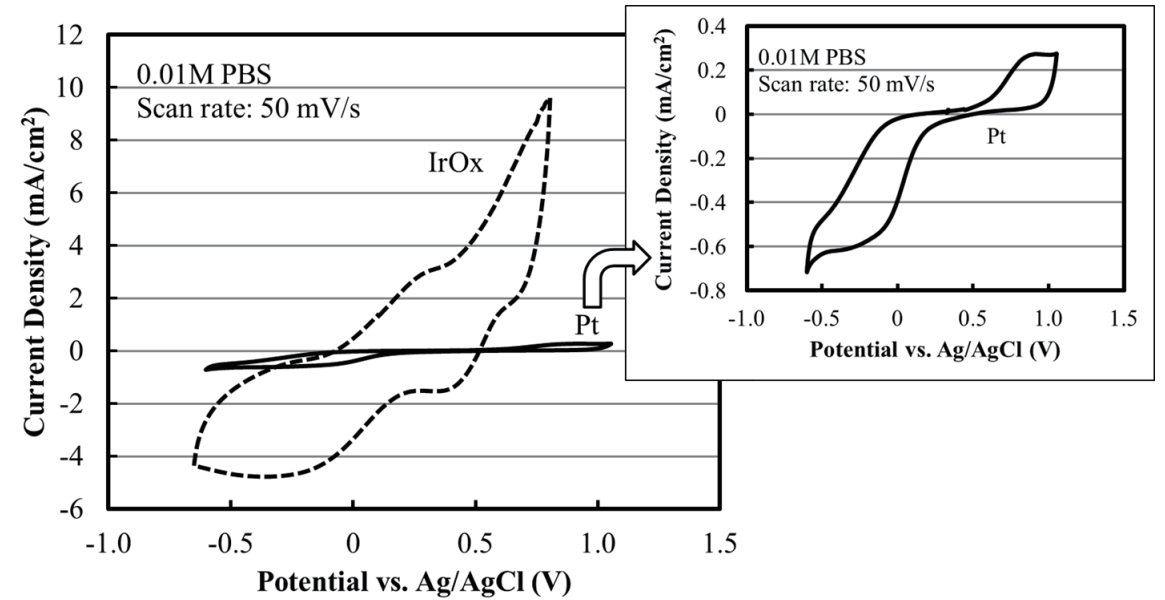

Fig. 7. Cyclic voltammogram of the planar electrodes. The dashed line shows the $\mathrm{CV}$ curve of the $\operatorname{IrO}_{x}$ electrode. The solid line shows the CV curve of the Pt electrode. The figure inset shows the enlarged CV curve of the Pt.

Table 2

CSCs of the planar electrodes.

\begin{tabular}{lc}
\hline & $\mathrm{CSC}\left(\mathrm{mC} / \mathrm{cm}^{2}\right)$ \\
\hline $\mathrm{IrO}_{x}$ & 110 \\
$\mathrm{Pt}$ & 9.4 \\
\hline
\end{tabular}




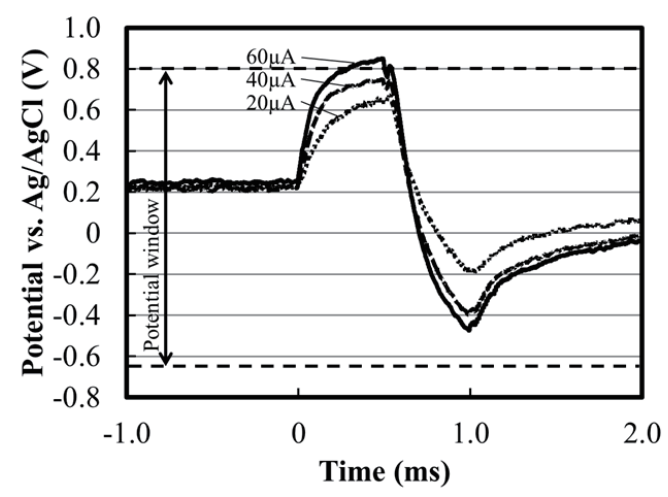

(a)

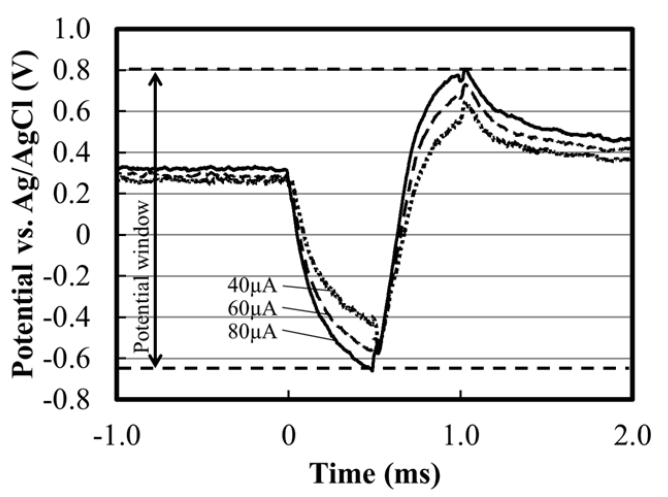

(b)

Fig. 8. Results of the transient potential measurement of the $\operatorname{IrO}_{x}$ electrode. (a) Anodic-first biphasic pulse. (b) Cathodic-first biphasic pulse.

Table 3

CICs of the planar electrodes.

\begin{tabular}{lcccc}
\hline & \multicolumn{2}{c}{ Maximum current $(\mu \mathrm{A})$} & \multicolumn{2}{c}{$\mathrm{CIC}\left(\mathrm{mC} / \mathrm{cm}^{2}\right)$} \\
\cline { 2 - 5 } & $\mathrm{AF}$ & $\mathrm{CF}$ & $\mathrm{AF}$ & $\mathrm{CF}$ \\
\hline $\mathrm{IrO}_{x}$ & 60 & 80 & 0.71 & 0.95 \\
$\mathrm{Pt}$ & 50 & 10 & 0.12 & 0.59 \\
\hline
\end{tabular}

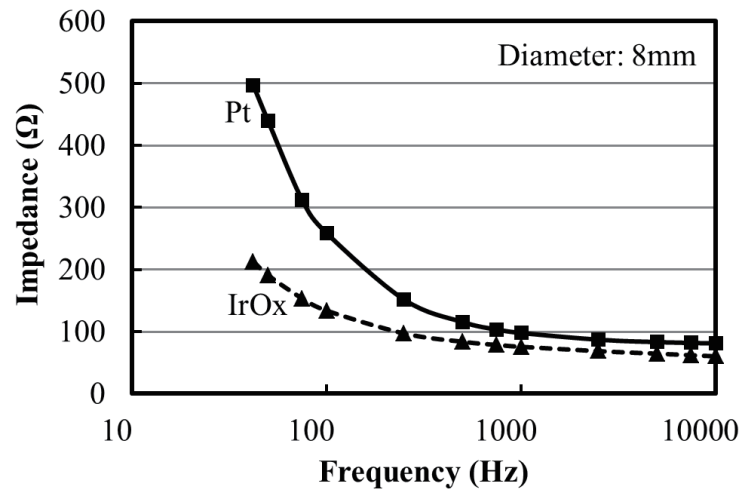

Fig. 9. Impedance of the $\mathrm{IrO}_{x}$ and $\mathrm{Pt}$ disc electrodes.

performance decreases along with the frequency increase. To demonstrate this, we measured the disc electrode impedance (diameter: $8 \mathrm{~mm}$ ) of $\mathrm{IrO}_{x}$ and Pt. Figure 9 shows that the $\mathrm{IrO}_{x}$ electrode exhibits a lower impedance than the Pt electrode at $1 \mathrm{kHz}$ or lower, but are similar above $1 \mathrm{kHz}$.

\subsection{Evaluation of performance improvement with the 3D structure}

Figure 10 shows the results of the CV measurement of the planar and 3D electrodes of $\operatorname{IrO}_{x}$. The larger CV loop of the 3D electrode compared with the planar electrode indicateds a higher CSC (Table 4). The total CSC (i.e., without area unit conversion) should be discussed in the case of the 3D electrode because of the difficulty of accurately measuring the geometric surface area. The total 


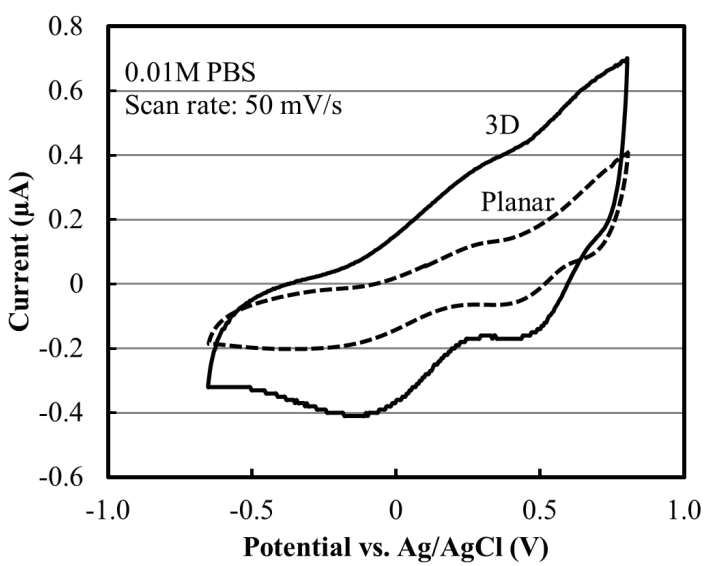

Fig. 10. Cyclic voltammogram of the planar and 3D electrodes. The solid line shows the CV curve of the 3D electrode. The dashed line shows the CV curve of the planar electrode.
Table 4

CSCs of the planar and 3D electrodes.

\begin{tabular}{lc}
\hline & CSC $(\mu \mathrm{C})$ \\
\hline Planar & 4.7 \\
3D & 9.1 \\
\hline
\end{tabular}

CSCs of the planar and 3D electrodes are 4.7 and $9.1 \mu \mathrm{C}$, respectively. The 3D electrode shows a 1.9 times higher performance than the planar electrode.

Figure 11 shows the results of the transient potential measurements of the planar and 3D electrodes. The noise level of measured potential of 3D electrodes was higher than that of planar electrodes. In our experiment, measured potential sometimes became noisy. The noise phenomena were not related to the shape of the electrodes, i.e. 3D or planar. Since the number of measured electrodes was limited, we could not measure typical properties in low noise. Although detailed discussion after measurement of a large number of electrodes is required, we can estimate the typical properties of the electrodes even if the number of measured electrodes is limited. Table 5 shows the maximum allowable current and CICs without unit area conversion. A comparison with the planar electrode shows that the 3D electrode has double the performance with the CF pulse and four times the performance with the AF pulse.

The enhancement of the electrode performance obtained by introducing the $3 \mathrm{D}$ structure is clearly demonstrated. However, while the surface area increased more than four times, the amount of improvement by the 3D structure is insufficient. One of the reasons for this may be the noneffective area of the charge transfer between the electrode surface and the electrolyte, which is caused by the non-uniform electric field generated by the 3D structure.

\subsection{Ex vivo evaluation}

Ex vivo experiments using an extracted pig eyeball are performed with STS configuration as one of the demonstrations. The surgery performed is similar to that reported in Ref. 23. A scleral pocket is first formed before inserting the $3 \mathrm{D} \mathrm{IrO}_{x}$ electrode. A Pt counter electrode is then inserted into the vitreous body. The three-electrode method is used following the measurement using the PBS described in the previous section. Figure 12(a) shows the experimental setup of the ex vivo $\mathrm{CV}$ measurement. Figure 12(b) presents the experimental setup of the ex vivo measurement of the 
Anodic-first
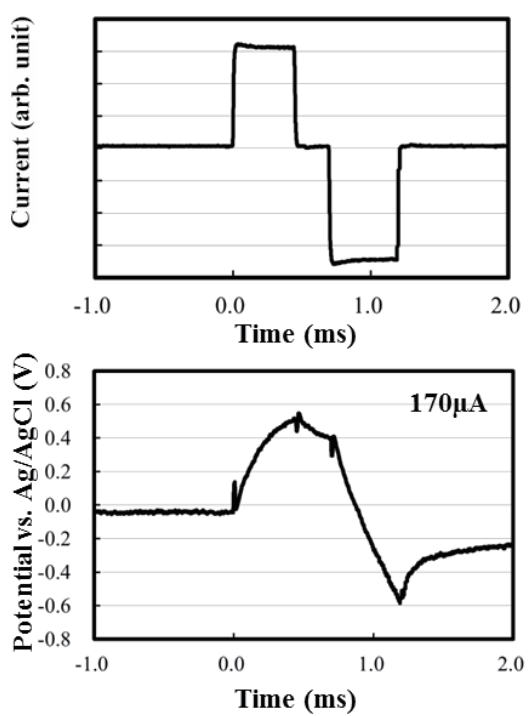

\section{Planar electrode}

3D electrode

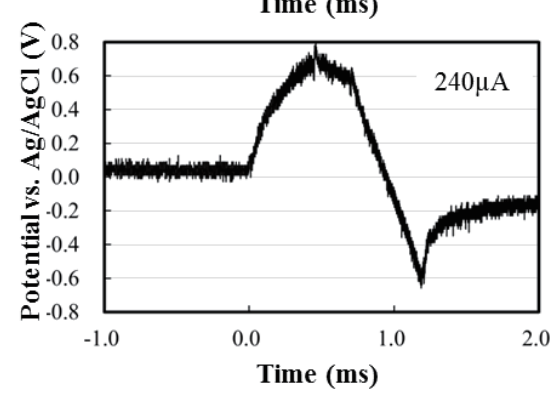

Cathodic-first
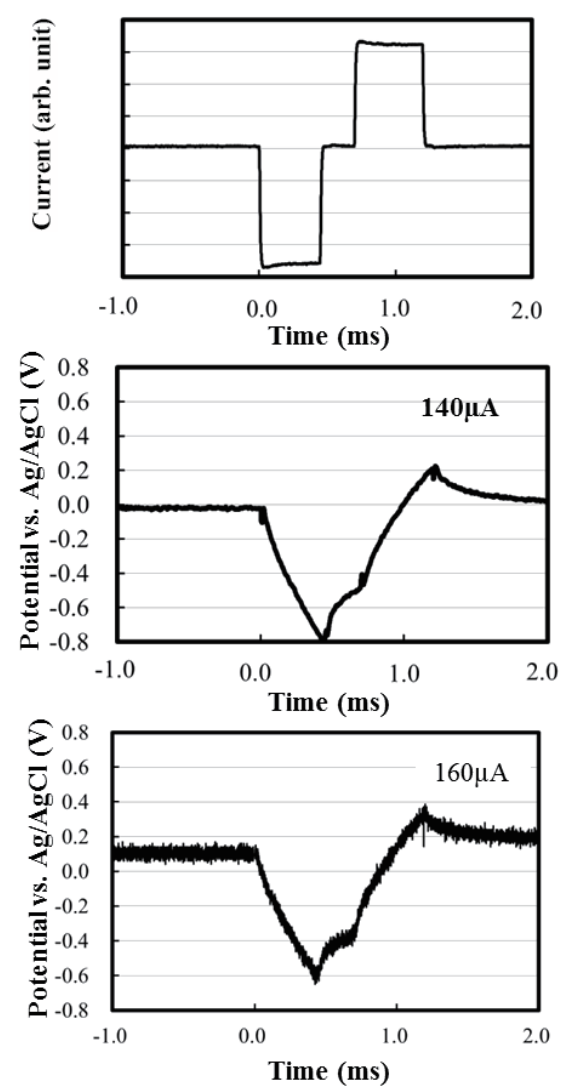

Fig. 11. Results of the transient potential measurements of the planar and 3D electrodes. The top row shows the waveforms of the applied pulse current. The middle row shows the transient potentials of the planar electrode. The bottom row shows the transient potentials of the $3 \mathrm{D}$ electrode. The left column presents the results with the anodic-first pulse, whereas the right column shows the results with the cathodic-first pulse.

Table 5

Maximum allowable current and CICs of the planar and 3D electrodes.

\begin{tabular}{lcccc}
\hline & \multicolumn{2}{c}{ Maximum current $(\mu \mathrm{A})$} & \multicolumn{2}{c}{$\mathrm{CIC}\left(\mathrm{mC} / \mathrm{cm}^{2}\right)$} \\
\cline { 2 - 5 } & $\mathrm{AF}$ & $\mathrm{CF}$ & $\mathrm{AF}$ & $\mathrm{CF}$ \\
\hline Planar & 170 & 140 & 0.03 & 0.04 \\
3D & 240 & 160 & 0.10 & 0.06
\end{tabular}

transient potential. A syringe with a needle primed with PBS is used to measure the electrolyte potential (i.e., vitreous body). The needle is inserted into the vitreous body. Subsequently, an Ag/ $\mathrm{AgCl}$ electrode is dipped into the PBS of the syringe.

\subsubsection{Ex vivo CV measurement}

The ex vivo $\mathrm{CV}$ measurement is then performed. The measurement parameters are as follows: scanning range of the work electrode potential: $-0.65-0.8 \mathrm{~V}$; scan rate: $50 \mathrm{mV} / \mathrm{s}$. Figure 13 shows 


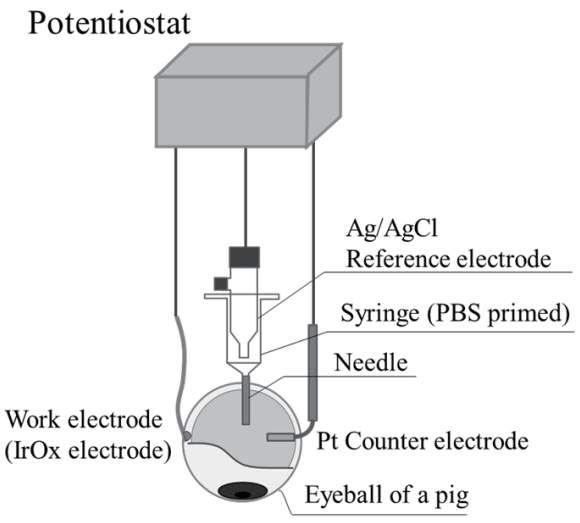

(a)

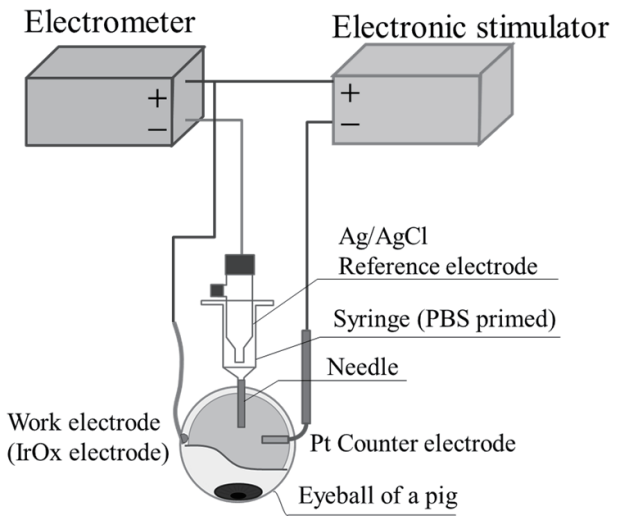

(b)

Fig. 12. Experimental setup of the ex vivo evaluation. (a) CV measurement. (b) Transient potential measurement.

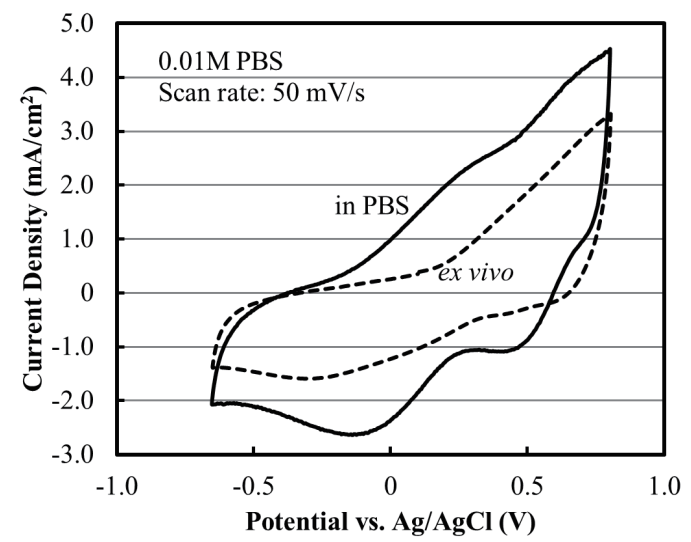

Fig. 13. Cyclic voltammogram of the 3D electrode. The solid line shows an ex vivo CV curve. The dashed line shows the CV curve in the PBS.

the $\mathrm{CV}$ measurement results. The $\mathrm{IrO}_{x} 3 \mathrm{D}$ electrode functions under the implantation. A CV curve with PBS, which is explained in Sect. 3.3, is also re-plotted in Fig. 13. The ex vivo redox peaks are decreased, whereas the fundamental trends of the ex vivo and PBS CV curves are similar. Table 6 shows the CSCs calculated from the CV curves. The ex vivo CSC is decreased to $76 \%$ of the CSC in the PBS. One of the possible reasons for the degradation is the lower amount of electrolytes at the electrode-tissue interface. The other possible reason is the insufficient circulation of electrolytes caused by eyeball extraction.

\subsubsection{Ex vivo measurement of the transient electrode potential}

An ex vivo measurement of the transient electrode potential is performed to evaluate the charge injection performance with the pulse current used for retinal stimulation. The same pulse parameters employed in the measurement using PBS described in Sect. 3.3 are used. Figure 14 shows the measurement results. The measured results in the PBS, which are similar to those in Fig. 11, are also shown again for comparison. The 3D electrode could inject charges when the 
Table 6

Ex vivo CSC and PBS CSC.

\begin{tabular}{lc}
\hline & CSC $\left(\mathrm{mC} / \mathrm{cm}^{2}\right)$ \\
\hline Ex vivo & 46.2 \\
In PBS & 60.6 \\
\hline
\end{tabular}

Anodic-first

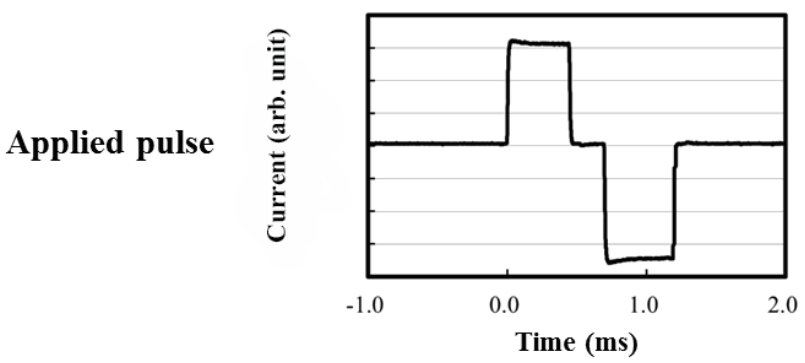

in PBS
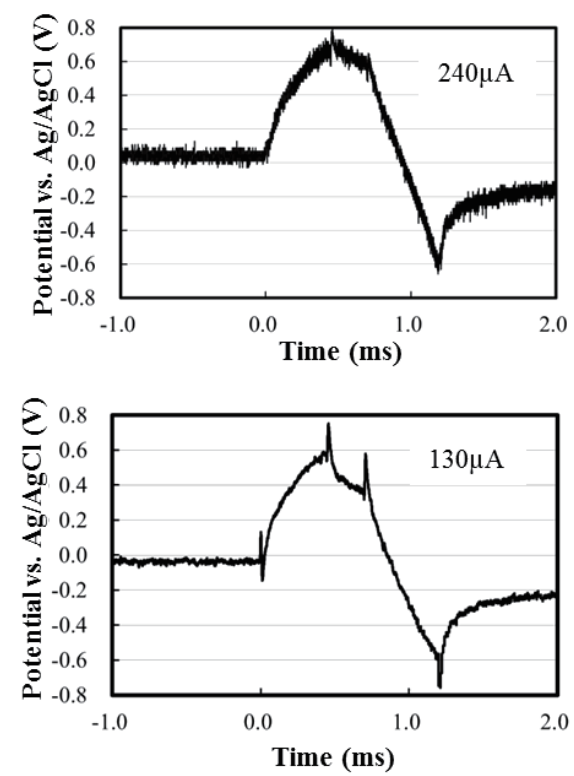

Cathodic-first
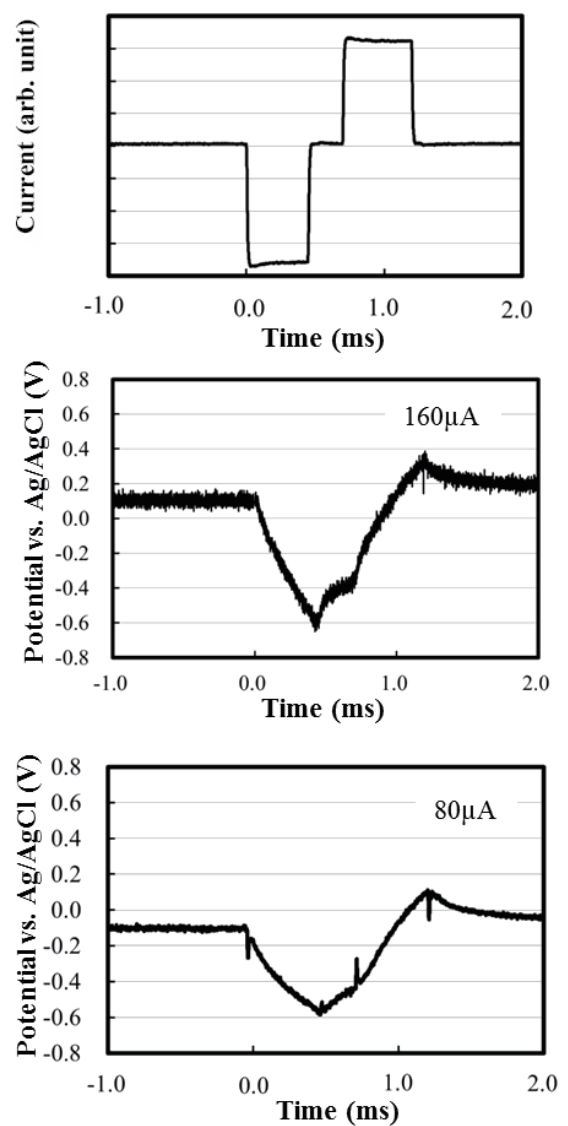

Fig. 14. Results of the transient potential measurement of the 3D electrode. The top row shows the applied pulse current waveforms. The middle row shows the transient potentials in the PBS. The bottom row shows the ex vivo transient potentials. The left column shows the results with the anodic-first pulse, whereas the right column presents the results with the cathodic-first pulse.

pulse current is applied under implantation. The maximum allowable currents in the PBS are 240 and $160 \mu \mathrm{A}$ with the $\mathrm{AF}$ and $\mathrm{CF}$ biphasic pulses, respectively. These values are 130 and $80 \mu \mathrm{A}$ with the AF and CF biphasic pulses, respectively, under implantation. Table 7 shows the CICs calculated from the results. The CIC under implantation is degraded to half of its value in the PBS. The degradation mechanism may be similar to that in the CSC degradation described in the previous section. Although the electrode performance is degraded under implantation, the electrode maintains a satisfactory performance for the stimulation. As one example, the stimulus threshold is $0.039 \mathrm{mC} / \mathrm{cm}^{2}{ }^{2(24)}$ Therefore, the fabricated electrode has ample margin for safe stimulation. 
Table 7

Maximum allowable current and CICs of the 3D electrode.

\begin{tabular}{lcccc}
\hline & \multicolumn{2}{c}{ Maximum current $(\mu \mathrm{A})$} & \multicolumn{2}{c}{$\mathrm{CIC}\left(\mathrm{mC} / \mathrm{cm}^{2}\right)$} \\
\cline { 2 - 5 } & $\mathrm{AF}$ & $\mathrm{CF}$ & $\mathrm{AF}$ & $\mathrm{CF}$ \\
\hline Ex vivo & 130 & 80 & 0.42 & 0.26 \\
In PBS & 240 & 160 & 0.77 & 0.51 \\
\hline
\end{tabular}

\section{Conclusion}

A high-performance electrode is required for use in the next-generation retinal prosthesis to miniaturize an electrode that will achieve high-resolution vision restoration. This paper reports on the fabrication and evaluation of high-performance electrodes by combining an introduced 3D structure and a high-performance material as a surface coating. The planar $\mathrm{IrO}_{x}$ and Pt electrodes are fabricated to evaluate the effectiveness of the high-performance material. The fabricated electrodes are characterized through electrochemical measurements. Consequently, the higher performance of $\mathrm{IrO}_{x}$ is quantitatively demonstrated as the CSC and the CIC. Introducing the 3D structure enlarges the electrode surface area, which is another approach to enhancing the electrode performance. A bump-shaped $\mathrm{IrO}_{x}$ electrode is fabricated. The electrochemical properties of this electrode are then compared with those of the planar electrode. The effectiveness of utilizing the 3D structure is clearly shown. The electrode performance under implantation is the most important consideration for practical use. Therefore, an ex vivo evaluation using an extracted pig eyeball is performed. A comparison with those in the PBS shows that the CSC and the CIC under implantation are degraded because the implanted environment is harsher than that in the PBS. This is an ideal situation to show a higher performance. The charge injection to the biological tissue is demonstrated using a pulse current similar to that in practical stimulations, although the performances are degraded.

We successfully demonstrate the proposed approach that uses $\mathrm{IrO}_{x}$ and the $3 \mathrm{D}$ structure to enhance the electrochemical performance. First, we use a stud bump to form the 3D structure. Other 3D structures, such as pillars and holes, may further enhance the performance in a future study. Micrometer or sub-micrometer pillars and holes can be fabricated using photolithographic resin, such as SU-8. The 3D resin structure and surface coating by $\mathrm{IrO}_{x}$ may be suitable for fabricating a miniaturized, high-performance electrode.

\section{Acknowledgements}

This work was supported by the Japanese-Taiwanese Cooperative Program on "Bioelectronics" and "Biophotonics" from the Japan Science and Technology Agency (JST).

\section{References}

1 K. Stingl, K. U. Bartz-Schmidt, D. Besch, C. K. Chee, C. L. Cottriall, F. Gekeler, M. Groppe, T. L. Jackson, R. E. MacLaren, A. Koitschev, A. Kusnyerik, J. Neffendorf, J. Nemeth, M. A. Naser Naeem, T. Peters, J. D. Ramsden, H. Sachs, A. Simpson, M. S. Singh, B. Wilhelm, D. Wong, and E. Zrenner: Vision Res. 111 (2015) 149.

2 T. Tokuda, Y. Takeuchi, Y. Sagawa, T. Noda, K. Sasagawa, K. Nishida, T. Fujikado, and J. Ohta: IEEE Trans. Biomed. Circuits Syst. 4 (2010) 445. 
3 T. Noda, K. Sasagawa, T. Tokuda, Y. Terasawa, H. Tashiro, H. Kanda, T. Fujikado, and J. Ohta: Electron. Lett. 48 (2012) 1328.

4 T. Noda, K. Sasagawa, T. Tokuda, H. Kanda, Y. Terasawa, H. Tashiro, T. Fujikado, and J. Ohta: Sens. Mater. 26 (2014) 637.

5 T. Noda, K. Sasagawa, T. Tokuda, Y. Terasawa, H. Tashiro, H. Kanda, T. Fujikado, and J. Ohta: Sens. Actuators, A 211 (2014) 27.

6 M. Monge, M. Raj, M. H. Nazari, H. Chang, Y. Zhao, J. D. Weiland, M. S. Humayun, Y. Tai, and A. Emami: IEEE Trans. Biomed. Circuits Syst. 7 (2013) 747.

7 A. Y. Chow, V. Y. Chow, K. Packo, J. Pollack, G. Peyman, and R. Schuchard: Arch. Ophthalmol. 122 (2004) 460.

8 B. Ilic, D. Czaplewski, P. Neuzil, T. Tanczyks, J. Blough, and G. J. Maclay: J. Mater. Sci. 35 (2000) 3447.

9 Y. Terasawa, H. Tashiro, Y. Nakano, K. Osawa, M. Ozawa, T. Noda, T. Tokuda, J. Ohta, and T. Fujikado: Invest. Ophthalmol. Visual Sci. 55 (2014) 1803.

10 H. Kanda, T. Morimoto, Y. Terasawa, Y. Nakano, K. Nishida, and T. Fujikado: Invest. Ophthalmol. Visual Sci. 54 (2013) 1046.

11 E. Slavcheva, R. Vitushinsky, W. Mokwa, and U. Schnakenberg: J. Electrochem. Soc. 151 (2004) 226.

12 S. F. Cogan, J. Ehrlich, T. D. Plante, A. Smirnov, D. B. Shire, M. Gingerich, and J. F. Rizzo: J. Biomed. Mater. Res., B 89 (2009) 353.

13 M. Patan, T. Shah, and M. Sahin: Conf. Proc. IEEE Engineering in Medicine and Biology Society 1 (2006) 890.

14 S. Venkatraman, J. Hendricks, Z. A. King, A. J. Sereno, S. R. Burns, D. Martin, and J. M. Carmena: IEEE Engineering in Medicine and Biology Society 19 (2011) 307.

15 S. Yao, M. Wang, and M. Madou: J. Electrochem. Soc. 148 (2001) 29.

16 C. C. Mayorga Martinez, R. E. Madrid, and C. J. Felice: Sens. Actuators, B 133 (2008) 682.

17 Y. Lu, T. Wang, Z. Cai, Y. Cao, H. Yang, and Y. Y. Duan: Sens. Actuators, B 137 (2009) 334.

18 K. H. Kondro, L. Ouattara, A. Trokourey, and Y. Bokra: Bull. Chem. Soc. Ethiop. 22 (2008) 125.

19 C. M. Nguyen, S. Rao, X. Yang, S. Dubey, J. Mays, H. Cao, and J. C. Chiao: Sensors 15 (2015) 4212.

20 Y. L. Pan, T. Noda, K. Sasagawa, T. Tokuda, and J. Ohta: IEEJ Trans. Electr. Electron. Eng. 8 (2013) 310.

21 Y. L. Pan, T. Noda, K. Sasagawa, T. Tokuda, H. Kanda, T. Fujikado, and J. Ohta: J. Phys.: Conference Series (JPCS) 3 (2012) 1.

22 A. Hung, I. B. Goldberg, and J. W. Judy: Implantable Neural Prostheses 2, eds. Zhou, David, Greenbaum, and Elias (Springer, Berlin, 2010) pp. 191-216.

23 T. Noda, T. Fujisawa, R. Kawasaki, H. Tashiro, H. Takehara, K. Sasagawa, T. Tokuda, and J. Ohta: 37th Annu. Int. Conf. IEEE Engineering in Medicine and Biology Society, Aug. 27, 2015, MiCo, Milano Conference Center, pp. 3355-8.

24 Y. T. Yang, P. K. Lin, C. Wan, W. C. Yang, L. J. Lin, C. Y. Wu, and C. C. Chiao: Invest. Ophthalmol. Visual Sci. 52 (2011) 9353. 